\title{
A comparison of forgetting rates in frequency discrimination and recognition
}

\author{
DOUGLAS L. HINTZMAN \\ University of Oregon, Eugene, Oregon \\ and \\ LEONARD D. STERN \\ Eastern Washington University, Cheney, Washington
}

\begin{abstract}
In two experiments on memory for pictures, a frequency-discrimination task was used to determine whether recognition decisions display slower forgetting than do discriminations among frequencies greater than 0. Experiment 1 compared frequency discriminations of 1-0 (recognition), 2-1, 4-1, and 4-2 and tested retention over an interval of 1 week. Experiment 2 added another recognition condition (2-0) and extended retention to 2 weeks. Neither experiment showed evidence for differential forgetting. The outcome is consistent with the hypothesis that the information underlying recognition memory and memory for frequency is qualitatively the same.
\end{abstract}

Are judgments of presentation frequency and judgments of recognition memory based on the same kind of remembered information? Intuitively, it seems they may not be-especially if the to-be-remembered items were initially unfamiliar. With such materials, a recognition judgment could be based simply on the feeling that the test item is familiar, whereas an accurate frequency judgment seems to require access to information of a more specific and qualititatively different kind. We are concerned here with the question of whether a qualitative difference in the kind of information underlying these two tasks might be reflected in forgetting rates. Specifically, this study contrasts two hypotheses: (1) that forgetting is slower in recognition memory than in memory for frequency, and (2) that the two forgetting rates are the same.

Some theoretical flesh might be put on the first hypothesis by invoking the distinction between generic memory-for abstract, general knowledge-and episodic memory-for specific, autobiographical experiences. This basic distinction has been made under various labels by a number of authors (see Hermann, 1982). Memory for frequency, like that for other details of presentation (e.g., modality, context, recency) is inherently episodic. On the other hand, a feeling of familiarity, in the absence of awareness of such details, could be considered a reflection of generic memory. If information in episodic memory is more easily lost than that in generic memory, as Tulving $(1972,1983)$ suggested, then the forgetting rate shown by a particular task should depend on the

This material is based upon work supported by the National Science Foundation under Grant BNS-7824987. Requests for reprints should be sent to Douglas L. Hintzman, Department of Psychology, University of Oregon, Eugene, OR 97403. relative weights given by that task to generic and episodic information. Thus, if recognition memory relies more on the general feeling of familiarity and less on episodic information than does memory for frequency, then recognition performance should display slower forgetting than is shown by frequency discrimination. ${ }^{1}$

One can frame essentially the same argument in terms of types and tokens (cf. Anderson \& Bower, 1973). Suppose the first presentation of an unfamiliar item establishes in memory both a type node (to represent the item) and a token node (to stand for that particular occurrence of the item), and that subsequent repetitions simply create additional token nodes. Finding either a type node or a token node in memory should be sufficient for recognition; but accurate memory for frequency requires retrieval of tokens. Obviously, in this special case, the type-token distinction corresponds to the generic-episodic distinction, and if one assumes in addition that type nodes are more resistant to forgetting than are token nodes, then recognition should decay more slowly than memory for frequency.

Theoretical support for the second hypothesis-that forgetting rates in the two tasks should be the same-can be found in Underwood's (1971) proposal that recognition memory is based on the frequency attribute and in the multiple-trace theory of memory proposed by Hintzman (1976; Hintzman, Grandy, \& Gold, 1981). A preliminary report of work with a simulation model of the latter theory can be found in Hintzman (1984). According to this theory, each repetition of an item does essentially the same thing that the first presentation does: It establishes its own memory trace. Thus, there is no type-token or generic-episodic distinction; and the difference between recognition and frequency discrimination is purely a quantitative one. That is, determining 
that something happened at least $\mathrm{N}$ times is basically the same, whether $\mathbf{N}$ is one or greater than one. If the loss of information in the individual traces of an item is independent of the number of such traces, then (as simulations confirm) the rates of forgetting in recognition and in frequency discrimination should be the same.

Two problems need to be dealt with if forgetting rates are to be compared. The first is that the circumstances in which subjects study and are tested should be as similar as possible across conditions. This will avoid confounding the manipulation of interest with other factors that might influence forgetting. In the present study, this requirement is easily satisfied, since a test of recognition memory is a special case of frequency discrimination. In both experiments, a forced-choice frequency-discrimination task was used, with frequency comparisons and retention intervals manipulated within subjects. The recognition condition was represented by the $1-0$ discrimination in Experiment 1 and by $1-0$ and 2-0 discriminations in Experiment 2.

The second problem is that the slopes of two forgetting curves falling at different performance levels should not be compared directly, because of possible distortion by floor and ceiling effects. The ideal solution to this problem would be to equate initial levels of performance in the two conditions somewhere below the ceiling. But this is extremely difficult to do (cf. Underwood, 1954, 1964), and it is often impossible without introducing confoundings with extraneous variables (e.g., number of learning trials or presentation rate) that could conceivably affect forgetting rate. The problem can, however, be dealt with through systematic variation. That is, one can deliberately vary performance level within one or both of the conditions that are to be compared. In this way, two families of forgetting curves are generated, and (providing the range of variation in at least one is sufficient), differential forgetting will be reflected in the crossing of the two sets of curves. The method of systematic variation is especially well suited to the present use of the frequency-discrimination task. We were able to choose several frequency comparisons that were known, from prior research, to yield immediatetest performance levels both above and below that for recognition. A slower rate of forgetting in recognition decisions should, therefore, be directly observable in the crossing or convergence of the curves.

There is one experiment in the literature similar to the two reported here. Underwood, Zimmerman, and Freund (1971) studied forgetting in a frequency-discrimination task, using a 7-day retention interval. Selective comparison of conditions in their Table 1 reveals no tendency for performance on pairs of frequency $>0$ items to decay more rapidly than that on pairs including a frequency $=0$ item. However, because the stimuli used by Underwood et al. were familiar words, the result is not really relevant to the question under consideration here. It may be supposed that generic memories already existed for the frequency $=0$ and frequency $>0$ words alike, and thus recognition-memory decisions could not differentially benefit from a redundant familiarity cue. The crucial feature distinguishing the present experiments from that of Underwood et al. (1971) was that unfamiliar stimuli (color vacation slides) were used. With such stimuli, frequency $=0$ items should lack both episodic and generic information-presumably a necessary condition for differential forgetting to occur.

\section{EXPERIMENT 1}

This experiment used two retention intervals $(30 \mathrm{~min}$ and 7 days) and four frequency-discrimination conditions: $1-0$ (the recognition condition), 2-1, 4-1, and 4-2. On the basis of previous research, performance in the 1-0 condition was expected to fall below that in the 4-1 condition, but above that in the $2-1$ and the $4-2$ conditions on the 30-min test.

\section{Method}

Design. The stimuli were color vacation slides presented at a 2-sec rate, in a list 160 positions long (two standard Kodak Carousel trays). The first 5 and last 5 positions were occupied by filler slides. The 150 experimental items were arranged in five overlapping blocks. Each block contained four frequency $=4$ items, four frequency $=2$ items, and six frequency $=1$ items. Repetitions were separated by 4 to 7 intervening items; and within a block, the ordering of conditions was random. Presentation of the successive slide trays was synchronized so that there was no extra intertray delay.

Each subject was given two frequency-discrimination tests: one $30 \mathrm{~min}$ after presentation of the list and one 7 days later. The items on the two tests were different. Either test consisted of 20 randomly ordered pairs of pictures, representing four frequency discriminations $(1-0,2-1,4-1$, and 4-2) from each of the five blocks of the presentation list.

Four rotations of items among conditions were achieved by (1) counterbalancing the ordering of the test lists given at the two delays, and (2) constructing two presentation lists in which the frequencies of the pictures that were paired for testing were interchanged (e.g., for Test Pair $A B$, if freq $(A)=1$ and freq $(B)=$ 4 in List 1 , then freq $(A)=4$ and freq $(B)=1$ in List 2 ). In this way, four groups of subjects were defined, across which were counterbalanced both retention interval and the relative frequencies of the members of each pair.

Subjects. Seventy-one paid subjects, recruited from the University of Oregon community, were tested. Testing was done in groups of 2 to 9 persons each. Four subjects failed to reappear for the second session, and their data were dropped. The data of 7 other randomly selected subjects were also dropped from the analysis, to equate at 15 the number receiving each of the four rotations of materials.

Procedure. The subjects were told they would see a series of vacation slides, some of which would be repeated, and that they were to study the slides for a memory test. The nature of the test was not revealed. The 160 -item list was then presented by a Carousel projector paced by a timer at a $2-\mathrm{sec}$ rate. Following this, the subjects were told that another experiment would be conducted before the memory test was given, and they spent about 30 min rating lists of nouns on semantic scales and being tested on memory for list membership of the nouns. Next, a test sheet for the first frequency-discrimination test was distributed. On it were 20 numbered "L-R" pairs. The subjects were told that, for each of the pairs of pictures, they were to decide which 


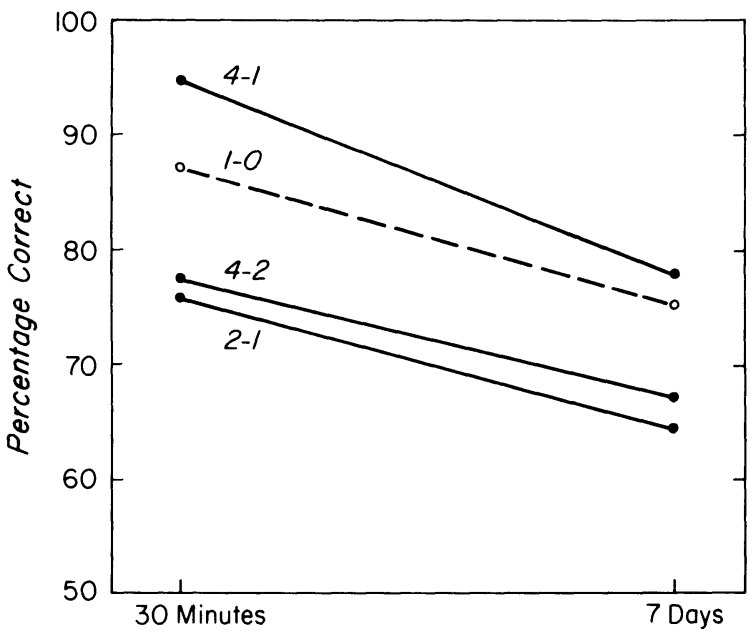

Retention Interval

Figure 1. Frequency-discrimination forgetting curves, Experiment 1 . The 1-0 discrimination (broken line) is the recognition condition.

member had occurred more often in the presentation list, and to circle either L (left) or R (right) accordingly. Test pairs were shown simultaneously, by two synchronized projectors, at an 8 -sec rate. After the test sequence, the subjects were told to return for a session scheduled 1 week later. The purpose of the second session was not specified. During the second session, the same test procedure was repeated, with the test list that had not been used during the first session.

\section{Results}

Correct-choice percentages in the four discrimination conditions are shown as a function of retention interval in Figure 1, in which each data point is based on 300 observations. As had been hoped, the method of systematic variation produced three frequency-discrimination curves that nicely bracketed that of the 1-0 (recognition) condition. The 1-0 curve did not cross any of the other three. Although the slope of the 4-1 curve was slightly greater than the slopes of the other conditions, the slopes of the 1-0,4-2, and 2-1 curves were virtually the same. A planned-comparisons ANOVA on arcsinetransformed scores confirmed this observation. The interaction between retention interval as one variable and $1-0$ versus the other three conditions as the other variable was not significant $[F(1,59)=0.04]$. Thus, the prediction that the recognition condition would be forgotten more slowly than the other frequency discriminations was not supported.

\section{EXPERIMENT 2}

Since Experiment 1 led to acceptance of the null hypothesis, a second experiment was designed to include several modifications intended to improve the potential for uncovering the predicted interaction: (1) The retention interval was lengthened to 2 weeks. (2) List length and test length were increased so that the number of observations per subject was at least doubled for every condition. (3) The total number of recognition observations was made the same as the number of frequency $>0$ observations. This was done by adding a $2-0$ condition and making the $1-0$ condition twice as large as the others.

\section{Method}

Design. The color slides were presented in a list 320 positions long (four Carousel trays). The 288 experimental items occupied the middle 72 positions of each slide tray, while the first 4 and last 4 positions contained filler slides. Altogether, the experimental items included 32 frequency $=4$ items, 48 frequency $=2$ items, and 64 frequency $=1$ items. The 288 experimental slides were ordered randomly with the sole restriction that a given picture could not immediately follow itself. Initially, pictures were assigned to three sets, determined by the number of available slides of each. Sixty-four pictures for which there were at least five copies were assigned to the 4-2 and 4-1 conditions. These were paired randomly to make 16 pairs in either condition. Another 64 for which there were at least three copies were assigned to the $2-1$ and $2-0$ conditions in a similar manner; and a final 64 for which there were two copies were assigned to the 1-0 condition, making two "dummy conditions" of 16 pairs each (one condition of 32 pairs). Four versions of the presentation sequence were generated by keeping the picture pairings for the test always the same, but swapping frequency assignments within a pair and switching pairs between the two condition assignments (e.g., 4-2 and 4-1) within a set.

As in Experiment 1, each subject was given two frequencydiscrimination tests. Either test included half of the pairs assigned to each condition-a total of 48 randomly ordered picture pairs. The order of the test lists was counterbalanced, yielding a total of eight groups of subjects (2 test orders $\mathrm{x} 4$ rotations).

Subjects. Eighty-six subjects, serving for extra credit in undergraduate psychology classes at the University of Oregon, were recruited. Of these, 9 failed to appear for the second session and 1 failed to properly follow instructions. For purposes of analysis, the data of 12 other randomly selected subjects were dropped to equate (at 8 ) the number in each of the eight groups.

Procedure. Presentation and testing were done much as in Experiment 1. Exceptions were that (1) there were three brief delays during presentation for slide-tray changes, (2) the interval between presentation and the beginning of Test 1 was about 4 min, during which subjects filled out self-addressed postcard reminders of the second session, (3) the presentation rate during testing was 5 sec per pair, and (4) the interval between the first and second sessions was 14 days. The postcards were mailed to the subjects 2 to 3 days before the second session.

\section{Results}

Correct-response percentages are shown in Figure 2, in which each 1-0 data point represents 1,024 observations and each of the others represents 512. Again, performance in the 1-0 condition fell below that in the 4-1 and above that in the 4-2 and the 2-1 conditions: and again the 1-0 curve was essentially parallel to the other curves. The 2-0 and 4-1 curves were virtually identical. A planned-comparisons ANOVA on the arcsine-transformed proportions showed a difference between Test 1 and Test $2[F(1,63)=180.0, p<.001]$, but the contrast between recognition (1-0 and 2-0 combined) and the other three conditions did not interact significantly with delay $[F(1,63)=0.02]$. Thus, the failure of Experi- 


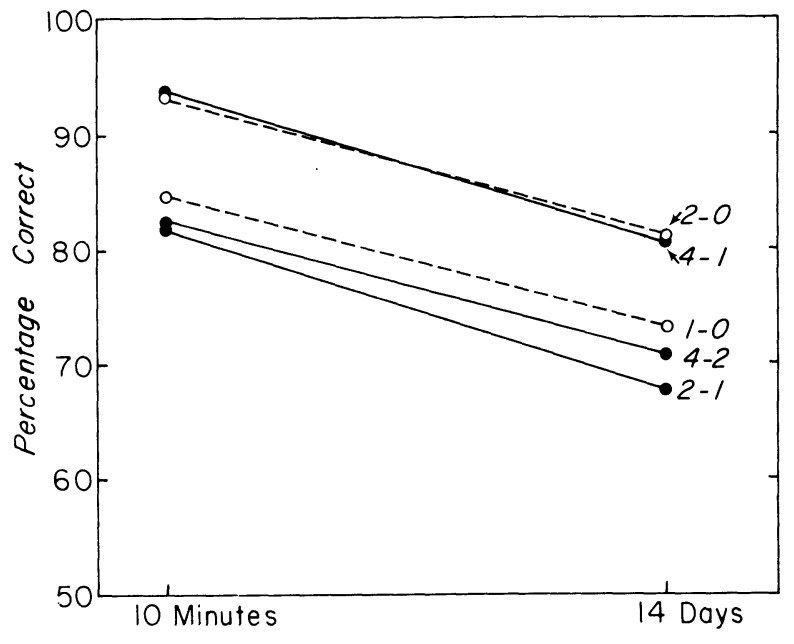

Rentention Interval

Figure 2. Forgetting curves for Experiment 2. Broken lines represent the recognition conditions.

ment 1 to reveal differential forgetting was completely confirmed by Experiment $2 .^{2}$

\section{DISCUSSION}

The basic question of interest here is whether recognition judgments and judgments of relative frequency are based on qualitatively different kinds of information. To the extent that the two hypothetical types of information are characterized by different forgetting rates, the answer appears to be no. Experiment 1 produced a visible but statistically unreliable interaction in the predicted direction; and even less evidence for the predicted interaction was found in the more powerful Experiment 2.

Of course, several arguments could be made against this conclusion: Perhaps retention intervals on the order of several months are necessary for the difference in forgetting rates to appear; or perhaps generic memories, the hypothetical source of the added familiarity cue in recognition, require several repetitions to become established. Lacking a well-articulated theory of episodic and generic memory and their interaction, however, such ad hoc objections are difficult to evaluate.

A further possibility is that, by embedding the recognition conditions in a frequency-discrimination task, we induced in our subjects a strategy of ignoring or not retrieving familiarity information and basing all judgments solely on episodic memory. In counterargument to this objection, it may be noted that, in Experiment 2, fully half the test items were old-new pairs, and it seems unlikely that all subjects would ignore information that is relevant to recognition decisions in such a situation. Furthermore, the forgetting rates in both experiments were not particularly fast. Insofar as we can tell, they were about what previous recognition-memory data would suggest.

Obviously, our null results leave several theoretical questions unanswered. Recognition and frequency decisions may both be based entirely on episodic information, or both entirely on familiarity, or both on the same mixture of the two; or, alternatively, two qualitatively different kinds of information, whose forgetting rates are the same, may be involved. Such speculation aside, the present results, together with those of Underwood et al. (1971), demonstrate that the loss of frequency information from memory is quite slow. Furthermore, the simple pattern of the data suggests a simple explanation. The data are consistent with a theory that assumes that the information underlying judgments of frequency and recognition memory is qualitatively the same. And the strikingly parallel forgetting curves suggest, in addition, that forgetting rate is independent of frequency. That is, the increments (or traces) left by successive repetitions are all lost at the same rate.

\section{REFERENCES}

Anderson, J. R., \& Bower, G. H. (1973). Human associative memory. Washington, DC: Winston.

Hermann, D. J. (1982). The semantic-episodic distinction and the history of long-term memory typologies. Bulletin of the Psychonomic Society, 20, 207-210.

Hintzman, D. L. (1976). Repetition and memory. In G. H. Bower, (Ed.), The psychology of learning and motivation (Vol. 10). New York: Academic Press.

HintzMAN, D. L. (1984). MINERVA 2: A simulation model of human memory. Behavior Research Methods, Instruments, \& Computers, 16, 96-101.

Hintzman, D. L., Grandy, C. A., \& Gold, E. (1981). Memory for frequency: A comparison of two multiple-trace theories. Journal of Experimental Psychology: Human Learning and Memory, 7, 231-240.

Mandle R, G. (1980). Recognizing: The judgment of previous occurrence. Psychological Review, 87, 252-271.

Tulving, E. (1972). Episodic and semantic memory. In E. Tulving \& W. Donaldson (Eds.), Organization of memory. New York: Academic Press.

Tulving, E. (1983). Elements of episodic memory. Oxford: Oxford University Press.

UNDE RWOOD, B. J. (1954). Speed of learning and amount retained: A consideration of methodology. Psychological Bulletin, 51, 276-282.

UNDE RWOOD, B. J. (1964). Degree of learning and the measurement of forgetting. Journal of Verbal Learning and Verbal Behavior, 3, 112-129.

UNDERWOOD, B. J. (1971). Recognition memory. In H. H. Kendler \& J. T. Spence (Eds.), Essays in neobehaviorism. New York: Appleton-Century-Crofts.

Unde rwood, B. J., Zimmerman, J., \& Freund, J. S. (1971). Retention of frequency information with observations on recognition and recall. Journal of Experimental Psychology, 87, 149-162.

\section{NOTES}

1. Mandler (1980) proposed a special role for familiarity in recognition memory. But in sharp contrast to the above argument (and to our intuition), he assumed that the decay of familiarity is relatively fast.

2. Some cautions are in order for those who might want to draw conclusions from comparisons of the relative levels of the obtained curves, either between or within Figures 1 and 2 . First, pictures were rotated through conditions only as appropriate to the purpose of the experiments; thus, the picture pairs contributing to all the curves were not the same. Second, the subjects in Experiment 1 were paid, and this may be one reason why the longer retention interval used in Experiment 2 produced no more forgetting than was found in Experiment 1.

(Manuscript received for publication April 2, 1984.) 